JIM 06050

\title{
Simultaneous flow cytometric detection of antibodies against platelets, granulocytes and lymphocytes
}

\author{
Kees Sintnicolaas ${ }^{1}$, Wim de Vries ${ }^{2}$, Reinier van der Linden ${ }^{2}$, Jan Willem Gratama ${ }^{2}$ \\ and Reinder L.H. Bolhuis ${ }^{2}$ \\ ${ }^{1}$ Blood Transfusion Service, and ${ }^{2}$ Department of Immunology, Dr. Daniel den Hoed Cancer Center, Rotterdam, The Netherlands
}

(Received 1 February 1991, revised received 30 April 1991, accepted 21 May 1991)

We present a time-saving and objective flow cytometric immunofluorescence assay for the simultaneous detection of antibodies against platelets, granulocytes or lymphocytes using a reconstituted mixture of these cell populations. Platelets, granulocytes and lymphocytes could be distinguished on the basis of their forward (FSC) and sideways (SSC) light scattering properties plotted on scales of 4 log orders. After setting FSC/SSC gates around the platelets, granulocytes and lymphocytes, the reactivity of the sera with the cell populations was determined by histogram analyses of immunofluorescence for each gate. The flow cytometric assay of reconstituted cell mixtures showed a strong, positive correlation with a reference microscopic immunofluorescence assay of separate cell suspensions. The reproducible procedures for the isolation and staining of the cells and the electronic stability of the flow cytometer permitted the use of the same gate and marker settings throughout the experiments. Consequently, the entire analysis of data stored in list mode could be performed using a keystroke, so that time consuming and subjective manual analyses were avoided.

Key words: Flow cytometry; Platelet; Granulocyte; Lymphocyte; Immunofluorescence; (Antibody)

\section{Introduction}

Antibodies against platelets, granulocytes and/or lymphocytes are important in immunohematology, e.g., in autoimmune hematological disease and in platelet transfusion therapy. The

Correspondence to: K. Sintnicolaas, Dr. Daniel den Hoed Cancer Center, Groene Hilledijk 301, 3075 EA Rotterdam, The Netherlands.

Abbreviations: BSA, bovine serum albumin; FSC, forward light scatter; GIFT, granulocyte immunofluorescence test; LIFT, lymphocyte immunofluorescence test; PBS, phosphatebuffered saline; PFA, paraformaldehyde; PIFT, platelet immunofluorescence test; PRP, platelet rich plasma; RT, room temperature; SSC, sideways light scatter. demonstration of autoantibodies on platelets and/or granulocytes may be valuable in establishing the autoimmune nature of thrombocytopenia or granulocytopenia (Verheugt et al., 1977; Von dem Borne et al., 1980). In platelet transfusion therapy, serological tests for the demonstration of alloantibodies against HLA antigens and/or platelet-specific antigens may establish alloimmunization as the cause of platelet refractoriness; such tests may also be used as a platelet crossmatch to select compatible platelet donors for alloimmunized patients (Freedman et al., 1984; Sintnicolaas et al., 1987).

Direct and indirect immunofluorescence techniques allow the detection of autoantibodies and/or alloantibodies to platelets, granulocytes 
or lymphocytes. The assays are routinely performed on suspensions of blood cells containing single, i.e., separated, purified cell populations (platelets, granulocytes or lymphocytes). Flow cytometry enables these cell populations to be distinguished in a mixed suspension on the basis of their different light scattering properties. The application of flow cytometry to the detection of antibodies against platelets, granulocytes and lymphocytes would permit the simultaneous detection of antibodies against those cells after electronic gate setting. We present here a simple and rapid immunofluorescence assay for the detection of autoantibodies and alloantibodies to platelets, granulocytes and lymphocytes that is performed using a reconstituted cell suspension and flow cytometric analysis.

\section{Materials and methods}

\section{Preparation of cell suspensions}

For screening sera for the presence of antibodies against platelets, granulocytes or lymphocytes, a cell panel derived from five randomly chosen donors was routinely used. Platelet-rich plasma (PRP) was obtained from EDTA-anticoagulated blood by centrifuging at $400 \times g$ for $10 \mathrm{~min}$. All centrifugation steps were carried out at $20^{\circ} \mathrm{C}$ using a temperature controlled centrifuge (Rotixa RP, Hettich, F.R.G.). The PRP was centrifuged at $1200 \times \mathrm{g}$ for $20 \mathrm{~min}$ to obtain a platelet pellet. The platelets were washed twice in a solution of $0.027 \mathrm{M} \mathrm{Na}$-EDTA in $0.013 \mathrm{M}$ phosphatebuffered $0.145 \mathrm{M} \mathrm{NaCl}$, pH 7.4 containing $0.2 \%$ bovine serum albumin (EDTA-PBS-BSA). To prepare granulocyte and mononuclear cell suspensions, EDTA-PBS-BSA was added to the 4-6 $\mathrm{ml}$ of cell suspension that remained after removal of PRP to give a final volume of $10 \mathrm{ml}$. To this suspension, $0.5 \mathrm{ml}$ of methylcellulose solution ( $2 \%$ in PBS), was added, mixed and incubated at $37^{\circ} \mathrm{C}$ for $30-45 \mathrm{~min}$ to allow gravity sedimentation. The leukocyte containing supernatant was harvested and the granulocytes were separated from the mononuclear cells by density centrifugation using Ficoll-Hypaque (Lymphoprep, $\mathrm{Ny}$ comed, Norway) at $1000 \times g$ for $20 \mathrm{~min}$. The interphase cells (mononuclear cells) were col- lected and washed once with EDTA-PBS-BSA. The erythrocytes in the cell pellet fraction were lysed by incubation with precooled $\mathrm{NH}_{4} \mathrm{Cl}$ for 5 min on melting ice. The remaining cells (granulocytes) were washed once with EDTAPBS-BSA. The three fractions were then washed once with EDTA-PBS without BSA, fixed with $1 \%$ paraformaldehyde (PFA) in PBS for $5 \mathrm{~min}$ at room temperature (RT) and washed twice using EDTA-PBS-BSA. After resuspension in EDTAPBS-BSA, the cell concentrations were adjusted to $150 \times 10^{9} / 1$ for platelets and $4.5 \times 10^{9} / 1$ for mononuclear cells and granulocytes. Finally, a mixed cell suspension was prepared by mixing equal volumes of the platelet, granulocyte and mononuclear cell suspensions.

\section{Preparation of $1 \%$ PFA in PBS}

A stock solution of $4 \%(\mathrm{w} / \mathrm{v})$ PFA was prepared by dissolving PFA in PBS while slowly heating to $70^{\circ} \mathrm{C}$. The solution was filtered and stored in the dark at $4^{\circ} \mathrm{C}$. The stock solution was used for a maximum of 4 weeks. A working solution of $1 \%$ PFA was prepared by dilution of the stock solution with PBS immediately before use.

\section{Indirect immunofluorescence test}

To $50 \mu \mathrm{l}$ of the mixed cell suspension $50 \mu \mathrm{l}$ of serum were added. Following incubation for 30 min at RT the cells were washed three times. 50 $\mu 1$ of a $1 / 40$ dilution of goat $\mathrm{F}\left(\mathrm{ab}^{\prime}\right)_{2}$-anti-human IgG conjugated with fluorescein isothiocyanate (FITC) (Dakopatts, Glostrup, Denmark) were added for $30 \mathrm{~min}$ at RT, followed by washing and flow cytometry. All tests were performed in duplicate.

\section{Flow cytometry}

Flow cytometry was performed on a FACScan (Becton and Dickinson Immunocytometry Systems (BDIS), San Jose, CA), equipped with an air-cooled argon ion laser emitting $488 \mathrm{~nm}$ at 15 $\mathrm{mW}$, a solid state silicon detector to detect forward light scatter (FSC) and three photomultiplier tubes to detect sideways light scatter (SSC), and green (FITC), orange-red and deep red fluorescence. Green fluorescence was collected through a $530 / 30 \mathrm{~nm}$ bandpass filter. The FSC, 
SSC and FITC fluorescence signals were amplified logarithmically. The data of 10,000 events were collected, stored in list mode and analyzed using Consort 30 software.

In the analyses, gates were set around the platelets, granulocytes and lymphocytes on the basis of their FSC and SSC characteristics. Histogram analyses of FITC fluorescence were performed for these 3 gates in which standard markers were placed to distinguish negative and positive events. These standard marker sets were chosen as follows. One positive and one negative serum was selected on the basis of their reactivity in the microscopic assay. These sera were then tested against platelets, granulocytes and lymphocytes from 20 donors in the flow cytometric and microscopic assays. For each gate, five different marker sets were arbitrarily chosen around the feet of the negative peaks obtained with the negative serum, and the percentages of positive cells calculated. These results were compared with those of the microscopic assays and the marker settings that gave maximum discrimination between microscopic negative and positive results were selected: PIFT, channels 200 and 10,000; GIFT, channels 460 and 10,000 ; and LIFT, channels 340 and 10,000. The standardized isolation and staining procedures of the cells permitted the use of identical gate and marker settings throughout the experiments. All tests were performed in duplicate and the mean values were used as the test results. A test result was scored 'positive' when the percentage of positive cells exceeded $30 \%$. That percentage resulted in the best positive correlation between the flow cytometric and microscopic results of PIFT, GIFT and LIFT assay (data not shown). A test result was rejected when the difference between the duplicate values exceeded $10 \%$.

\section{Microscopic immunofluorescence tests}

The platelet immunofluorescence test (PIFT), granulocyte immunofluorescence test (GIFT) and lymphocyte immunofluorescence test (LIFT) were performed as previously described (Decary et al., 1975; Verheugt et al., 1977; Von dem Borne et al., 1978). Cell suspensions of platelets, granulocytes and mononuclear cells were prepared as described above. Final cell concentrations were
$100 \times 10^{9} / 1$ for platelets and $1.5 \times 10^{9} / 1$ for granulocytes and mononuclear cells. All tests were performed in duplicate and all slides were independently read by two investigators. Fluorescence intensity was scored from negative to strongly positive, i.e.,,,-+++ to +++ .

\section{Patient and control sera}

Serum samples were harvested from whole blood which had been allowed to clot for $2 \mathrm{~h}$ at RT and stored at $-70^{\circ} \mathrm{C}$. Sera from multitransfused patients reacting with platelets, granulocytes and lymphocytes from more than $90 \%$ of random donors (MS sera) served as positive controls. Negative control sera were obtained from untransfused male blood donors. Control and test sera were always $A B O$ compatible with the test cells.

\section{Monoclonal antibodies}

For the identification of lymphocytes we used anti-Leu4/FITC (CD3, T lymphocytes) and antiLeu16/FITC (CD20, B lymphocytes) (BDIS). Granulocytes were identified using VIM-D5 (CD15), monocytes using My-4 (CD14) (Coulter Clone, Hialeah, FL), platelets using CLBthromb/1 (CD61) (Central Laboratory of the Dutch Red Cross Blood Transfusion Service, Amsterdam, The Netherlands), and erythrocytes using VIE-G4 (reactive with glycophorin A). VIM-D5 and VIE-G4 were kindly donated by Dr. W. Knapp (Institute for Immunology, Vienna University, Vienna, Austria). When unconjugated monoclonal antibodies were used, a second incubation was performed with goat anti-mouse IgFITC (Nordic). All antibodies and conjugates were diluted in EDTA-PBS-BSA and used at saturating concentrations.

\section{Results}

The use of a 4-order logarithmic scale for FSC and SSC permitted the simultaneous display of platelets, granulocytes and lymphocytes. For each cell population gates were set as shown in Fig. 1. The cell mixtures were immunophenotyped to analyze the composition of the gated cell populations (Table I). In the platelet gate (gate 1), 79\% 


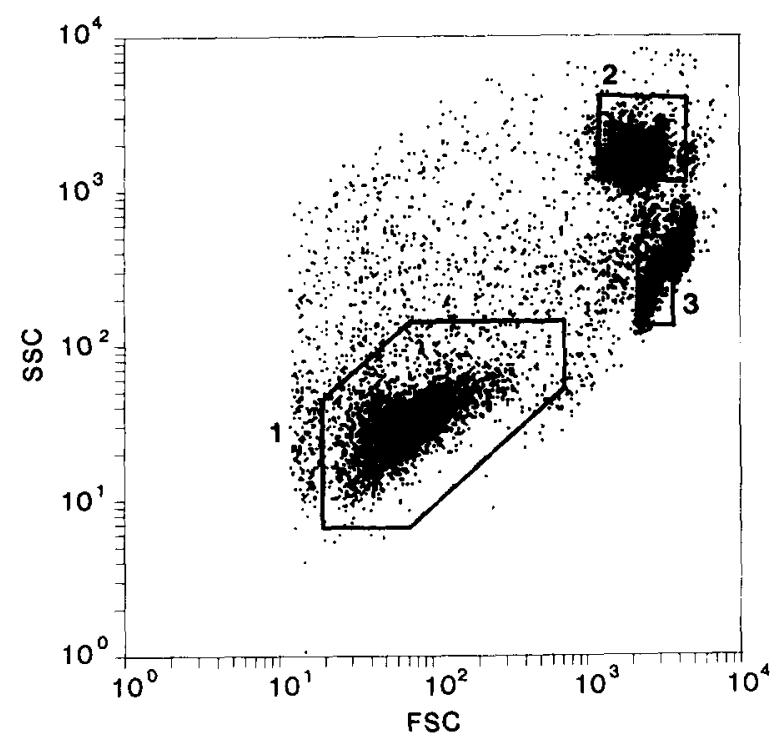

Fig. 1. FSC (horizontal axis)/SSC (vertical axis) dot plot of a reconstituted cell mixture. A 4 order logarithmic scale is used for both axes. The standard gates for platelets (gate 1), granulocytes (gate 2) and lymphocytes (gate 3) are shown. The cluster of events with FSC/SSC properties larger than lymphocytes represents the monocytes.

[3\%] (mean [SD] of five experiments) of the cells were positive for CD61; in the granulocyte gate (gate 2), $80 \%$ [10\%] of the cells were positive for CD15 and in the lymphocyte gate (gate 3), $84 \%$ [8\%] of the cells were positive for either CD3 or $\mathrm{CD} 20$. The purity of the cell populations selected by these gates was considered sufficient to permit detection of antibodies against the respective cell populations.

A cell mixture prepared from a random donor was incubated with a serum containing multispecific anti-HLA antibodies and a negative control serum (Fig. 2). Using the gate settings as described above, separate FITC fluorescence histograms were obtained for platelets, granulocytes and lymphocytes. Clear differences between the fluorescence intensities of the negative and positive sera were observed in all three cases. The cells in the granulocyte and lymphocyte gates of the negative control sample revealed a second peak of cells with intermediate fluorescence. Additional experiments (not shown) revealed that these intermediate fluorescent cells also stained positive with propidium iodide. Thus, the intermediate fluorescence of the granulocytes may have been caused by cell membrane damage. Two colour immunofluorescence analysis of mononuclear cell suspensions (not shown) revealed that the cells with low fluorescence were mainly $\mathrm{CD}^{+}$ (i.e., T lymphocytes) and that the cells with intermediate fluorescence were mainly $\mathrm{CD} 20^{+}$(i.e. lymphocytes) or $\mathrm{CD}_{14}{ }^{+}$(i.e., monocytes). Thus the intermediate fluorescence of the cells within the lymphocyte gate may result from (a) surface IgG on B lymphocytes and (b) aspecific-binding of serum IgG and/or conjugate to $\mathrm{Fc}$ receptors on monocytes present in the lymphocyte gate. The presence of cell populations with intermediate fluorescence led to an increase in the percentage of positive cells in the negative controls, but did not prohibit the detection of alloantibodies (see below).

To study whether or not the mixing procedure of the platelets, granulocytes and lymphocytes per se would influence the immunofluorescence signals, experiments were simultaneously performed using reconstituted cell mixtures and non-mixed cells, i.e., with separate suspensions of platelets, granulocytes and mononuclear cells

\section{TABLE I}

IMMUNOPHENOTYPING OF CELL POPULATIONS BY FLOW CYTOMETRY

\begin{tabular}{|c|c|c|c|c|}
\hline $\begin{array}{l}\text { Cell } \\
\text { population }\end{array}$ & Phenotype & $\begin{array}{l}\text { Platelet } \\
\text { gate (\#1) }\end{array}$ & $\begin{array}{l}\text { Granulocyte } \\
\text { gate (\#2) }\end{array}$ & $\begin{array}{l}\text { Lymphocte } \\
\text { gate (\#3) }\end{array}$ \\
\hline Platelets & CD61 & $79[3]^{a}$ & $4[5]$ & $2[4]$ \\
\hline Granulocytes & CD15 & $7[3]$ & $80[10]$ & $2[1]$ \\
\hline Monocytes & $\mathrm{CD} 14$ & $0[1]$ & $0[0]$ & $2[2]$ \\
\hline Lymphocytes & $\mathrm{CD} 3+\mathrm{CD} 20$ & $2[1]$ & $6[3]$ & $84[8]$ \\
\hline Erythrocytes & Glycophorin A & $0[1]$ & $2[2]$ & $0[0]$ \\
\hline
\end{tabular}

\footnotetext{
a Mean [1 standard deviation] $(n=5)$.
} 

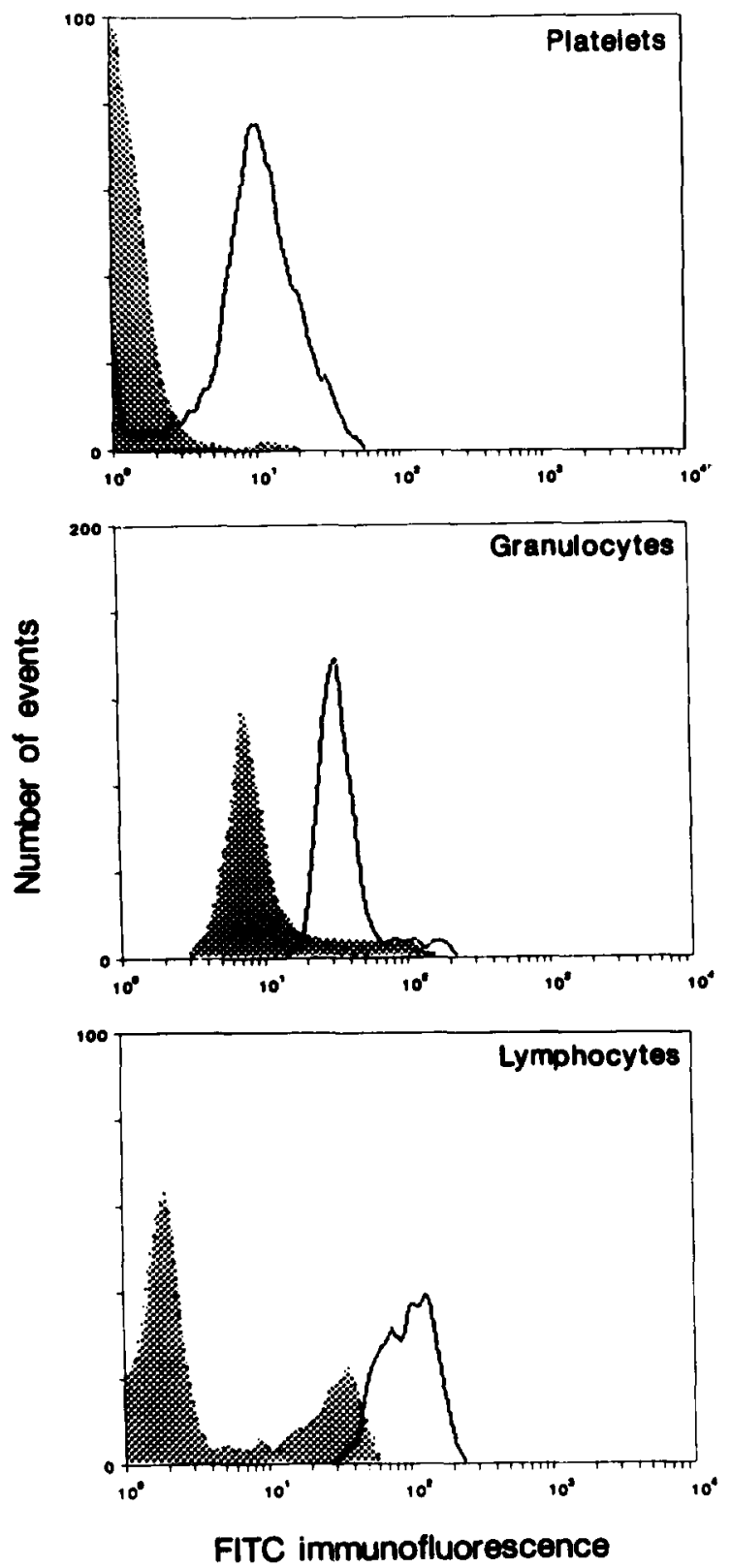

Fig. 2. Example of fluorescence histograms. On the horizontal axis FL1 (FITC fluorescence) is indicated on a log scale. On the vertical axis the number of events is given on a linear scale. Shaded curve: negative control serum. Open curve: positive control serum.

without further modification of the test. Three patient sera were selected that showed strong and selective reactivity against a single cell population (platelets or granulocytes or lymphocytes) in the conventional microscopic assay. These sera were tested in conjunction with a negative and a positive control serum against a cell panel derived from 5 randomly chosen blood group $\mathrm{O}$ donors. The results are shown in Fig. 3. For each serum and cell donor, the percentage of positive cells obtained using non-mixed cell supsensions was subtracted from the corresponding percentage obtained using reconstituted cell suspensions. The resulting differences (mean [SD]) were 4\% [5\%] ( $p=0.0003$; Wilcoxon signed rank test) for platelets, $2 \%$ [6\%] $(p=0.13)$ for granulocytes and $0 \%$ [6\%] $(p=0.57)$ for lymphocytes. The results obtained with mixed cells and separate cell suspensions were strongly and positively correlated (platelets, $r=0.99$; granulocytes, $r=0.98$; lymphocytes, $r=0.99$ ). Thus, mixing of the cells did not significantly affect the immunofluorescence signals obtained from granulocytes and lymphocytes, and led to a small but significant increase in the percentage of positive platelets.

Finally, flow cytometric analyses were compared with microscopic analyses on separate cell suspensions for the detection of serum alloantibodies (Table II). 24 sera from patients with hematological malignancies receiving platelet transfusion therapy were tested in both assays against a panel derived from five randomly chosen blood group $O$ donors. These experiments were performed on three occasions (eight sera and five cell donors per occasion). Flow cytometry and microscopy yielded identical scores for platelets in 108 of the 118 evaluable tests, granulocytes in 96 of 114 and lymphocytes in 100 of 117. An analysis of discrepant scores revealed that flow cytometry yielded significantly more positive reactions than microscopy for platelets and lymphocytes $(p=0.002$ and $p<0.001$ respectively). Thus, flow cytometry was more sensitive in the detection of antibodies against platelets and lymphocytes than microscopy. Flow cytometry and microscopy did not differ significantly in the detection of antibodies against granulocytes. In addition, the results were analyzed for each serum. A serum was scored reactive when cells of more than two out of five donors of the cell panel gave weakly positive reactions (microscopic score: + , flow cytometry $30-50 \%$ positive cells) or when cells of at least one donor of the panel gave 

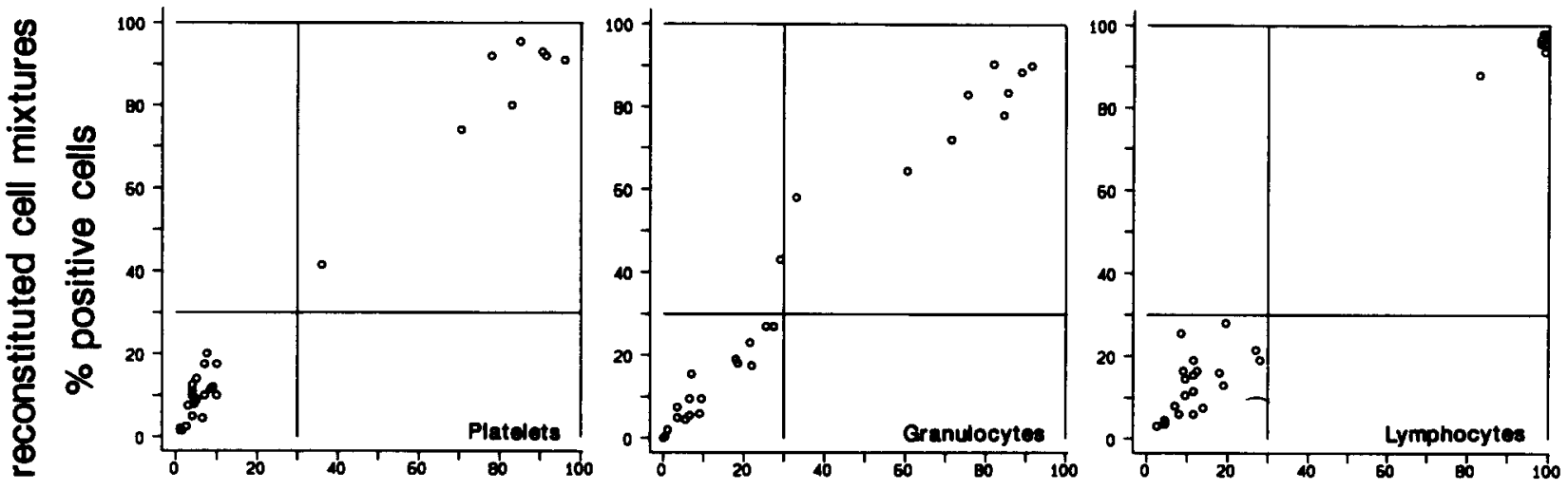

\section{separate cell suspensions: $\%$ positive cells}

Fig. 3. Comparison of the use of reconstituted cell mixtures with separate cell suspensions. Horizontal axis: percentage of positive cells using separate cell suspensions. Vertical axis: percentage of positive cells using reconstituted cell mixtures. The $30 \%$ lines on both axes indicate the cut-off values for discrimination between negative and positive test results (see materials and methods section).

strongly positive reactions (microscopic score ++ or +++ ; flow cytometry assay $>50 \%$ positive cells). For 20 of the 24 sera tested, identical scores were obtained. No antibodies were detected in 14 sera, HLA antibodies (i.e., antibodies reactive with platelets, granulocytes and lymphocytes) were detected in four sera and platelet specific antibodies (i.e., antibodies reactive with platelets only) were demonstrated in two sera. Discrepant results were found in four sera: three were reactive only in the flow cytometric assay (two sera were reactive with lymphocytes only; one serum with granulocytes only) and one serum reacted in the flow cytometric assay with platelets and lymphocytes and in the microscopic assay with granulocytes and lymphocytes.

\section{Discussion}

Initially, immunofluorescence microscopic tests were performed on separate cell suspensions, i.e., containing only platelets, granulocytes or lymphocytes and thus requiring separate examinations (Decary et al., 1975; Verheugt et al., 1977; Von dem Borne et al., 1978). Apart from being time consuming, a major disadvantage of microscopic tests is that the results are subject to observer bias. Recently, flow cytometric tests have been described for the detection of alloantibodies against platelets (Rosenfeld et al., 1986; Wang et al., 1989) and lymphocytes (Garovoy et al., 1983; Scornik et al., 1989). Also, the simultaneous detection of alloantibodies against granulocytes,

TABLE II

COMPARISON OF THE FLOW CYTOMETRIC IMMUNOFLUORESCENCE ASSAY APPLIED TO RECONSTITUTED CELL MIXTURES WITH THE MICROSCOPIC METHOD APPLIED TO SEPARATE CELL SUSPENSIONS

\begin{tabular}{|c|c|c|c|c|}
\hline $\begin{array}{l}\text { Flow } \\
\text { cytometric } \\
\text { assay }\end{array}$ & $\begin{array}{l}\text { Microscopic } \\
\text { method }\end{array}$ & Platelets & Granulocytes & Lymphocytes \\
\hline- & - & 87 & 82 & 82 \\
\hline+ & + & 21 & 14 & 18 \\
\hline- & + & $0 \quad p=0.002 *$ & $10 \longrightarrow$ n.s. & $0 \longdiv { p < 0 . 0 0 1 }$ \\
\hline+ & - & 10 & 8 & $17 \longrightarrow$ \\
\hline Not evaluab & & 2 & 6 & 3 \\
\hline
\end{tabular}

Key: $-=$ negative test result; $+=$ positive test result; $*=$ sign test; n.s. $=$ not significant. 
lymphocytes and monocytes, but not platelets, has been reported (Robinson et al., 1987). The assessment of the reaction pattern of a serum against three blood cell populations of a single donor is important since information about the specificity of antibodies can thus be obtained. For instance, anti-HLA antibodies are reactive with platelets, granulocytes and lymphocytes, whereas antibodies against platelet or granulocyte specific antigens react only with platelets or granulocytes, respectively.

We present here a flow cytometric method for performing simultaneous immunofluorescence tests on platelets, granulocytes and lymphocytes from a single donor for the detection of antibodies against these cells. The use of 4-order logarithmic scales for FSC and SSC signals permitted the identification of platelets, granulocytes and lymphocytes by standard FSC/SSC gate settings in reconstituted cell mixtures prepared from separate cell suspensions. The cell mixtures were reconstituted in such a way that a similar number of events was recorded in the three gates. Contamination of these gated cell populations with other cell types was $<20 \%$ in most cases. A potentially time-saving alternative to the use of reconstituted cell mixtures would be the use of buffy coat preparations in which the erythrocytes have been lysed. However, the concentrations of platelets, granulocytes and mononuclear cells may differ considerably from donor to donor, leading to variable ratios between cell types on the one hand and antibodies on the other. Hence, we have not explored this approach further. To discriminate between positive and negative cells in each gate, markers were set on the basis of the fluorescence histograms of the negative control serum, which were kept constant throughout the experiments.

Using sera exclusively reactive with platelets, granulocytes or lymphocytes we demonstrated that the reconstitution procedure did not lead to major changes in the immunofluorescence signals compared to the separate cell suspensions. A slight but significant increase in the percentage of positive platelets was observed which did not interfere with the detection of antibodies to platelets. A comparison between the flow cytometric assay applied to reconstituted cell mix- tures and the microscopic assay applied to separate cell suspensions revealed a greater sensitivity for the flow cytometric assay in detecting antibodies to platelets and lymphocytes, whereas both assays performed similarly in the detection of antibodies to granulocytes.

The standardized isolation and staining procedures of the cells and the electronic stability of the FACScan flow cytometer permitted unchanged gate and marker settings throughout the experiments. A major advantage of this situation was that the analysis and calculation of the results could be fully automated through a keystroke avoiding the time consuming and subjective adjustments of the gate and marker settings which would have required manual analysis.

\section{Acknowledgements}

We gratefully acknowledge Elly Fieret for her invaluable technical assistance and Johanna Dumon Tak-Garschagen for typing this manuscript.

\section{References}

Décary, F., Vermeulen, A. and Engelfriet, C.P. (1975) A look at HLA antisera in the indirect immunofluorescence technique test. In: Histocompatibility Testing 1975. Munksgaard, Copenhagen, p. 380.

Freedman, J., Hooi, C. and Garvey, M.B. (1984) Prospective platelet crossmatching for selection of compatible random donors. Br. J. Haematol. 56, 9.

Garovoy, M.R., Rheinschmidt, M.A., Bigos, M., Perkins, H., Colombe, B., Feduska, N. and Salvatierra, O. (1983) Flow cytometry analysis: a high technology crossmatch technique facilitating transplantation. Transplant. Proc. 15, 1939.

Robinson, J.P., Duque, R.E., Boxer, L.A., Ward, P.A. and Hudson, J.L. (1987) Measurement of antineutrophil antibodies by flow cytometry: simultaneous detection of antibodies against monocytes and lymphocytes. Diagn. Clin. Immunol. 5, 163.

Rosenfeld, C.S. and Bodensteiner, D.C. (1986) Detection of platelet alloantibodies by flow cytometry. Am. J. Clin. Pathol. 85, 207.

Scornik, J.C., Salomon, D.R., Richard, P.B.M., Howard, R.J. and Pfaff, W.W. (1989) Posttransplant antidonor antibodies and graft rejection. Transplantation 47, 287.

Sintnicolaas, K., Sizoo, W. and Bolhuis, R.L.H. (1987) Prospective selection of compatible platelet donors for alloimmunized patients by an ELISA platelet crossmatch 
only. In: C.Th. Smit Sibinga, P.C. Das and C.P. Engelfriet (Eds.) White Cells and Platelets in Blood Transfusion. Martinus Nijhoff Publishers, Boston, MA, p. 53.

Verheugt, F.W.A., Von dem Borne, A.E.G.Kr., Décary, F. and Engelfriet, C.P. (1977) The detection of granulocyte alloantibodies by an indirect immunofluorescence test. $\mathrm{Br}$. J. Haematol. 36, 533.

Von dem Borne, A.E.G.Kr., Verheugt, W.A., Oosterhof, F., Von Riesz, E., Brutel de la Rivière, A. and Engelfriet, C.P. (1978) A simple immunofluorescence test for the detection of platelet antibodies. Br. J. Haematol. 39, 195.
Von dem Borne, A.E.G.Kr., Helmerhorst, F.M., Van Leeuwen, E.F., Pegels, H.G., Von Riesz, E. and Engelfriet, C.P. (1980) Autoimmune thrombocytopenia: detection of platelet autoantibodies with the suspension immunofluorescence test. Br. J. Haematol. 45, 319.

Wang, G.X., Terashita, G.Y. and Terasaki, P.I. (1989) Platelet crossmatching for kidney transplants by flow cytometry. Transplantation 48, 959. 